\title{
Glassless Augmented Display for Public Signage
}

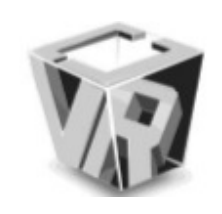

\author{
Hisataka SUZUKI ${ }^{1}$, Akihiko SHIRAI ${ }^{1}$, Kazuhisa YANAKA ${ }^{1}$
}

${ }^{1}$ Kanagawa Institute of Technology, Japan

Abstract - This project realizes spatial augmented reality in public. We developed Glassless Augmented Display(GAD) which can show multiple different images to different direction simultaneously. Spatial augmented reality allows audiences to watch their preferred images and audios on public signage. It is a highly applicable technology configured by a consumer-based $4 \mathrm{~K}$ liquid crystal display, superdirective speakers, a lenticular lens, and a multi-view image synthesizer implemented in a Unity application. We also proposed an application for GAD, which is called Simultaneous Spatial Shared Display (SSSD), which is implemented on Glassless Augmented Diaplay. SSSD enabled (1) simulmultiplex content display, (2) perspective correction, (3) without glasses and any sensing system, (4) directional audio for each content, and (5) attachable to conventional 2D display. These are used to display various contents, such as sports games and concerts, according to the movement of the audience.

Index Terms - multiplex imagery; shader technology; lenticular lens; signage; augmented display.

\section{Motivation: Spatial Augmented REALITY IN THE PUBLIC}

Public viewing on a large screen and/or digital signage has rapidly become popular in recent years. These public screens are usually placed in stadiums, parks, public halls, and restaurants, and are used to display various contents, such as sports games and concerts. Public viewing can be used commercially or non-commercially with or without advertisement in public transportation hubs, such as stations and airports. Public screens show information on departures and arrivals of trains and airplanes, as well as show advertisements and provide information to travelers. Such screens should display various information with static/motion pictures depending on the situation. These screens generally have a large, unspecific audience and includes persons of various ages and nationalities, and those who have physical disabilities. Providing different contents to different people simultaneously based on the observer's interests and attributes is difficult for a conventional display because it consists of no more than a screen. Therefore, we propose a new system that can solve this problem by combining configured

\footnotetext{
E-mail: gadeshirai.la
}

glassless 3D display technology and superdirective speaker technology.

3D display devices are widespread nowadays, but no significant application, especially for public screens, exists. The proposed project is called "Glassless Augmented Display (GAD)", which features a new display system in which multiple virtual screens are produced on a single physical screen. This technology is based on current glassless 3D stereo graphics technology. It can present different types of information for many unspecified people at the same time by combining a $4 \mathrm{~K}$ display device with a lenticular lens plate and a multiview image synthesizer that runs on a graphics processing unit (GPU). GAD can present multiple channels simultaneously without the need for viewers to use a wearable device, such as stereo glasses. Information can be presented efficiently through various means, such as augmented reality and multi-language and/or multi-directional formats, in theaters, living rooms, and in public space. Viewing a completely different image is possible through viewing position interactivity.

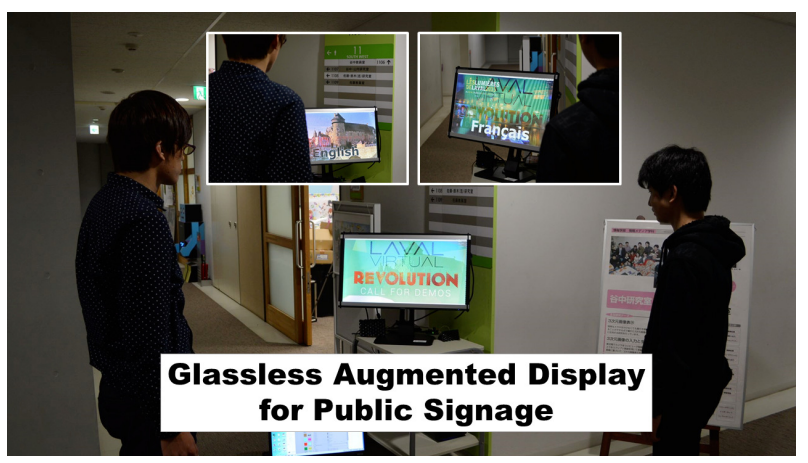

Figure 1: Prototype of proposing Glassless Augmented Display for public signage. Each viewer can see different images and audios from different directions.

\section{RELATED WORKS}

In 1692, Gaspar's drawing "Bois-Clair" had double portraits, which allow a viewer to see different angles(Antoine ). In the late 1930s, Vari-Vue successfully formed a multipleimage lenticular image; this development gave rise to the Vari-Vue company. During this time, Vari-Vue coined the term "lenticular"(Vari-vue ). 
Bimber and Rasker proposed Spatial Augmented Reality in 2005(Oliver and Ramesh 2005). The technology extends projector utilization, such as projection mapping. Invoked computing, which involves a configured projector, camera, parametric speaker, and steering based on Laval Virtual ReVolution 2011, is an ideal realization is needed for the environment in this system, and moving projectors and the target user must be alone or limited.

Suzuki et al. proposed multiplex-hidden technologies that can produce multiple channels on a screen, and these technologies continue to the fourth generation by using projectors and flat panels(Suzuki, Hsieh, and Shirai 2014). However, multiplex-hidden technology is based on polarization and color canceling. Audiences need a filter to choose their preferred contents. The idea of using autostereo display to realizespatial augmented reality is not new.

Fujitsu Ten, AVN7905HD (FUJITSU-TEN 2005), and Mercedes Benz SPLITVIEW (Mercedes-Benz ) are similar technologies that are applied to car navigation systems for two sides, such as the driver and assistant seats. Sharp Inc. proposed a triple view liquid crystal display(LCD) with a parallax layer to provide three viewing angles (SHARP ). In this system, different contents can be seen from the driver's seat, the assistant's seat, and the backseat. However, these techniques are not scalable because they focus on application to a car and are not always suitable for digital signage. Digital signage requires high resolution, interactive and/or moving pictures, a large screen with less cost, and the ability to be viewed by various audiences even in a bright space. Our challenge is to extend public displays virtually by using our scalable light field display(LFD) method to realize GAD for multidirectional viewers.

\section{METHOD}

The proposed system consists of a video subsystem and an audio subsystem, as shown in Figure 2. In the video subsystem, a number of virtual video channels are achieved by using a single real display. In the audio subsystem, many different sound waves are radiated from super-directivity parametric speakers to different directions.

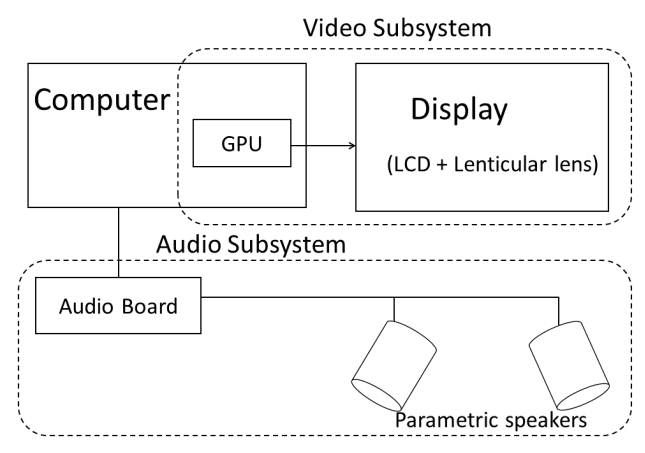

Figure 2: System configuration of GAD.

\section{III.I. VIDEO SUBSYSTEM}

Our proposed method involves configuring a LFD that consists of an LCD and a lenticular lens panel, as shown in Figure 3. A transparent acrylic plate of the foreground is used to fix the lenticular lens panel tightly.

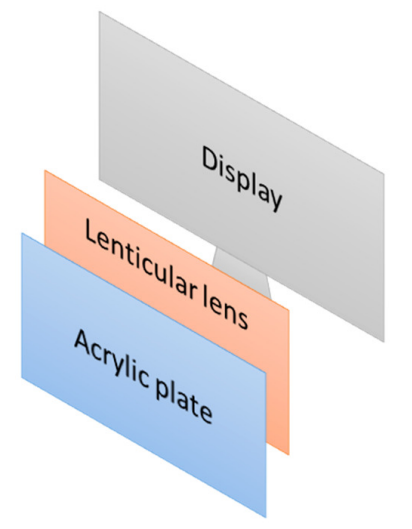

Figure 3: Display configuration of GAD.

LFD that uses an LCD is often used for autostereoscopic 3D display because a 3D image is bright and clear. In a 3D display, two or more images that are captured from slightly horizontally different camera positions are synthesized, and the resultant image, which is usually called a lenticular image, is obtained. A stereoscopic image is seen when the lenticular image is displayed on the LCD and observed through a lenticular lens placed just above the LCD. In a 3D display, each view corresponds to one of the images used to synthesize the lenticular image. Although the quality of the 3D image decreases when crosstalk occurs among different views, this decrease is not detrimental because views are usually mutually similar, especially in the case of multiview autostereoscopic displays.

Completely different contents-for example, a sports program, a music program, and a news program-are highly probable to be displayed in each view in the proposed system. If crosstalk occurs, then unrelated contents are mixed, thereby producing discomfort in users. A precise optical system is required to prevent crosstalk.

Figure 4 shows a sectional view of a simple conventional LFD using a lenticular lens, which is an array of semicylindrical lenses. Red, green, and blue subpixels are placed just below the flat side of the lens sheet. The light from each subpixel is almost a parallel beam because the focal length is usually almost equal to the thickness of the lens sheet. Figure 4 shows eight subpixels per lens pitch. Therefore, the number of the directions in which light travels is eight. Thus, the number of views is also eight. This system is called the integer view method because eight is an integer.

When off-the-shelf LCD and ready-made lenticular lens are combined to produce an LFD, the possibility that the ratio of the pixel pitch and the lens pitch is exactly an integer number by chance is almost zero. In most cases, it becomes a noninteger or a "fractional"value. Even in this 


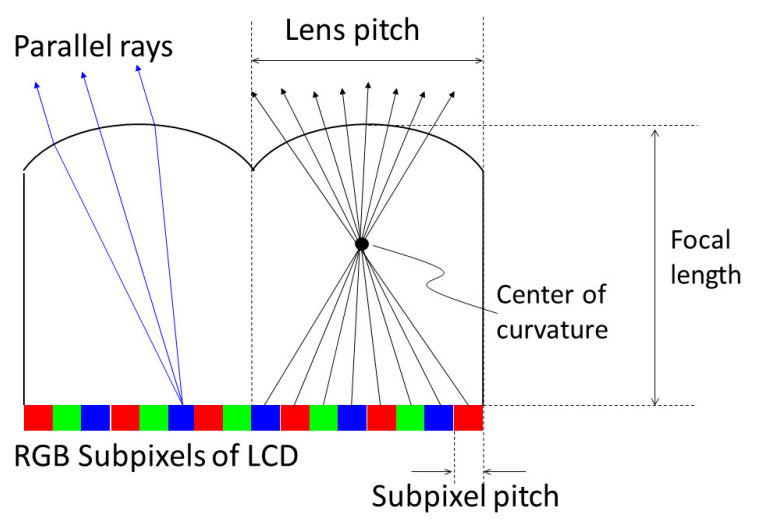

Figure 4: integrel view method.

case, an LFD can be produced by using Ishii's fractional view method (Ishii 2006). An attached lenticular panel provides a non-integer (fractional) light direction set from subpixels. Ishii's method was extended by Yanaka to cope with integral photography that has both horizontal and vertical parallax (Yanaka 2007) (Yanaka 2008).

The display system we adopted in this project is categorized as fractional view because a non-integer number of pixels are assumed to exist per lens pitch. The calculation amount is reduced substantially by replacing the timeconsuming ray tracing process from each subpixel to choose the most appropriate rendered image from a number of prerendered images. In the fractional view system, unsightly moire may occur because the lens pitch and the subpixel pitch do not have an integer ratio. Therefore, we slanted the lenticular lens panel against the LCD, as shown in Figure 5. Moire became less visible because it was transferred to a high-frequency band, and the subjective image quality was improved substantially.

Conventionally, the slanted lenticular system has been adopted in multi-view 3D display systems. The purpose of slanting is to increase the number of views in this case, and the angle of slanting has to be a specific angle that is obtained theoretically. The angle of inclination for the reduction of the moire has considerable acceptable width. We tilted the lenticular lens $10^{\circ}$ in the experiment, which will be described later.

Where

$$
H=\frac{O}{\cos (90-\theta)}
$$

$H$ stands for number of pixels per horizontal lens pitch and $O$ stands for number of pixels per original lens pitch (we describe about calculation of pixels per lens pitch later) show in Figure 5. The total number of views is assumed to be three in Figure 5 to ensure easy understanding of the figure, although the actual number of views is 12 in the experiment that will be described later. Given that the number of views is three, each semi-cylindrical lens is divided into three areas. The view that each subpixel indicates is de- cided by the area to which the center of the subpixel belongs. Numbers 1 to 3 on each subpixel show a view to which the subpixel belongs. High processing capability is required for real-time synthesis of plural video signals. We perform the synthesis by using a GPU.

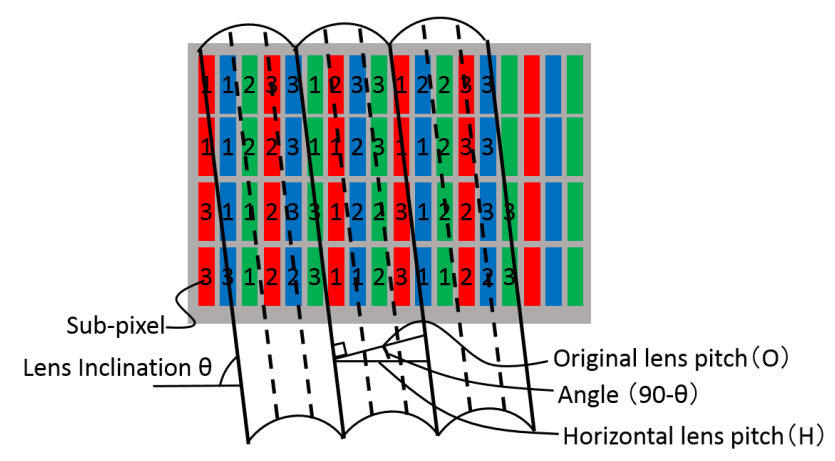

Figure 5: Slanted lenticular system for moire reduction.

To synthesize display image, we need to calculate where to put each input images. $H$ and lens inclination $\theta$ are parameter to calculate threshold value $T$ by equation 2 .

$$
T=\frac{\left(\frac{x}{3}-\frac{y}{\tan (\theta)}+H \times 100\right) f \bmod H}{H}
$$

In equation2, we added $H \times 100$ to avoid the negative number result of whole calculation.

Finally, we allocate input images by equation 3 . Subpixel $P_{(x, y)}$ is the brightness of the input image subpixel $C(n)_{(x, y)}$, where $i$ is determined by the equation 2 based on the value of $T$. Here it is noted that the unit of $x$ is a subpixel, whereas the unit of $y$ is a pixel.

$$
P_{(x, y)}= \begin{cases}C(1)_{(x, y)} & \left(0 \leq T<\frac{1}{n}\right) \\ C(2)_{(x, y)} & \left(\frac{1}{n} \leq T<\frac{2}{n}\right) \\ \vdots & \\ C(n-1)_{(x, y)} & \left(\frac{n-2}{n} \leq T<\frac{n-1}{n}\right) \\ C(n)_{(x, y)} & \text { (otherwise) }\end{cases}
$$

\section{III.II. Audio Subsystem}

In this system, aside from presenting different images and videos in different directions, the acoustic associated with these images and videos must also be presented. Therefore, we use parametric speakers, which have high directivity. Video and audio signals must be synchronized. Thus, they are output from the same PC by using Unity, which is a popular game engine.

\section{EXPERIMENT}

Figure 6 shows the installation of the system. In the experiment, audiences can see different visual contents sent to three directions and two different audios that accompany them. 


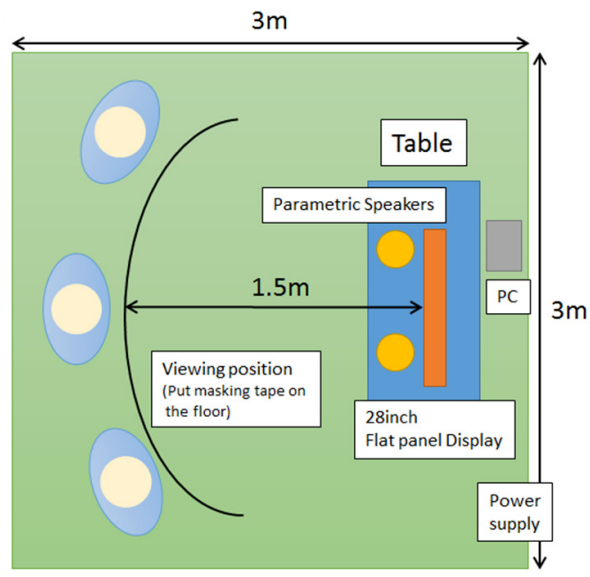

Figure 6: Installation of the system.

\section{IV.I. LCD AND LENTICULAR LENS}

In the experiment, IIYAMA ProLite B2888UHSU, which is a 28-inch 4K (3840x2160pixels) LCD, was used among various $4 \mathrm{~K}$ displays that are widely distributed in the market. A 15-line-per-inch plastic lenticular lens was used among products that are readily available in the market. The main specifications of the LCD and its relation to the lenticular lens are shown in Figure 7. The value of pixels per lens pitch in Figure 7 represents a number of pixels that covered by one lens.

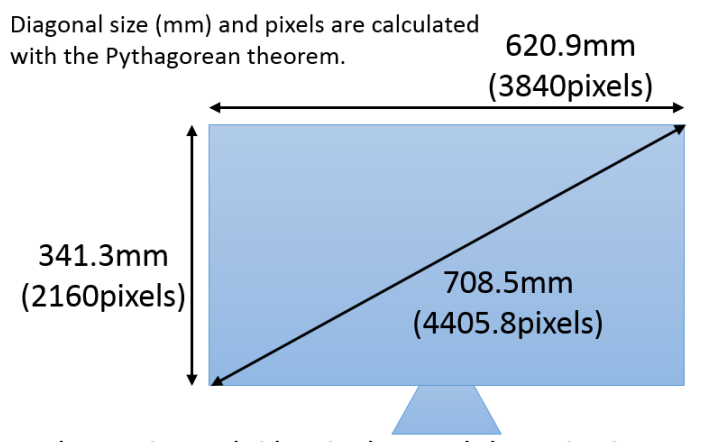

When using $15 \mathrm{lpi}$ lenticular, each lens size is $1.69 \mathrm{~mm}$.

$$
\text { Pixels per lens pitch is } \frac{1.69 \mathrm{~mm}}{0.1608}=10.5099
$$

Figure 7: Main specifications of the LCD and its relation to the lenticular lens.

\section{IV.II. Parametric Speakers}

Different sounds are played by multiple parametric speakers (Switch Science, SSCI-018425) turned to different directions. A sufficiently high directivity was achieved because mixing of sounds did not occur.

\section{IV.III. OVERAll Operation OF The System}

We created content to test the overall operation, as shown in Figure 8. A still image is visible when the screen is viewed from the center, and a French video and an English video are visible when the screen is viewed from the left and the right sides, respectively. Audios that are attached to the two videos can be heard without mixing. Proposed prototype can provide a multi-language signage for the public and it is helpful for international event and conference, as well as provide a visitor survey with three sides to share and visualize audience impressions on the site. The experiment proved that audiences can see different contents and audios played in three directions. The system can also provide interaction.

In our experiment, the audience stopped and viewed their preferred contents on their own. This behavior was unexpected because we had planned addition of interactivity will be extend in further step. However, it seems more natural user centered solution to see contents actively. Face recognition, gaze tracking, and/or visibility evaluation will be implemented.

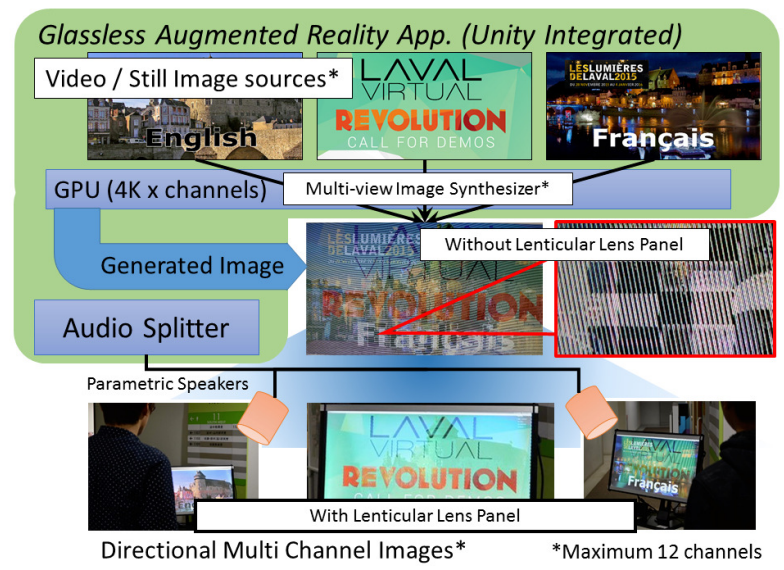

Figure 8: Glassless Augmented Display and system configuration.

\section{Application: Simultaneous Spatial SHARED DISPLAY}

We proposed an application for GAD, which is called SSSD, enabled (1) simul-multiplex content display, (2) perspective correction, (3) glassless without any sensing system, (4) directional audio for each content,and (5) attachment ability for conventional 2D display. In the video subsystem, which is built by Unity engine, 12 virtual video channels can be achieved using a single real display. In the audio subsystem, various sound waves are radiated from super-directive parametric speakers toward different directions.

Homographic transformation can correct perspective views (Jones et al. 2015) (Jagannatha and Jawahar 2005). We applied this homographic transformation to realize the perspective correction for all views and thus generate a more natural view for each direction of the viewers (Figure 9). 
Figure 10 shows a result from the left side but the audience can see a frame in rectangular shape.

Furthermore, this transformation is optimized to each view and does not affect the other views because of the simul-multiplex technology. The result of our approach is show as Figure 11.

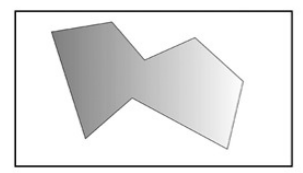

Original image

(a)

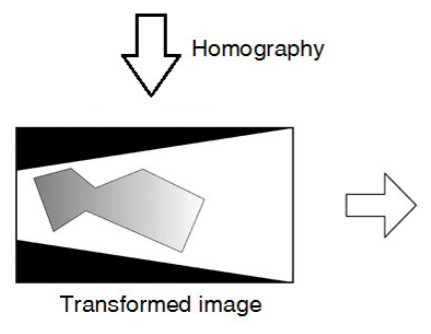

(c)

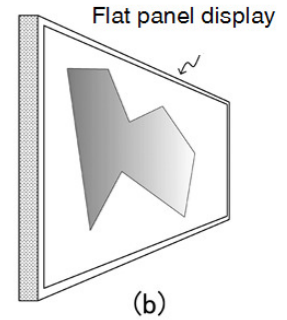

(b)

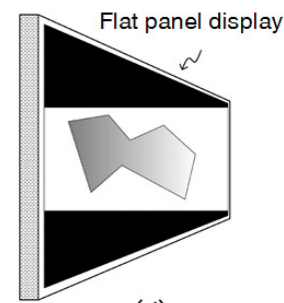

(d)
Figure 9: Homographic transformation for perspective correction.

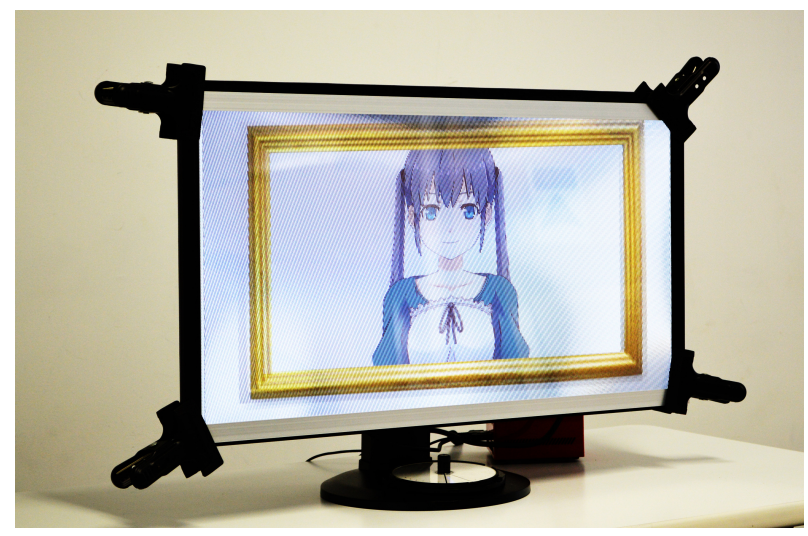

Figure 10: Example of perspective corrected left side image.

The proposed prototype can provide a multi-language signage for the public. This project is also helpful for international events and conferences, theater, stadium, traffic sign, size sensitive fine arts, billboard character animation, remote robot manipulation, multi-audience interactive media, augmented reality advertising and printing, etc. It can show future applications of ultra high resolution LCD and light field display (Figure 12).

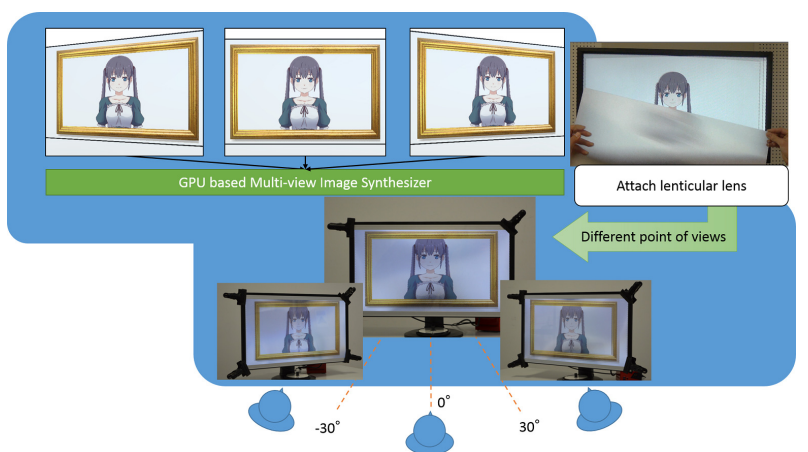

Figure 11: Result: each audience can see perspective view even they are placed at different position.

\section{Conclusion}

A new multimedia display system that consists of a multidirectional display and superdirective speakers was proposed, and its prototype was produced by using a high-resolution (4K) LCD and a lenticular lens. Different visual contents, and accompanying audios are played simultaneously, and each user can choose his/her preferred contents by walking left and right in front of the display to change viewing positions. The system is highly applicable because all software systems are integrated into a Unity application.

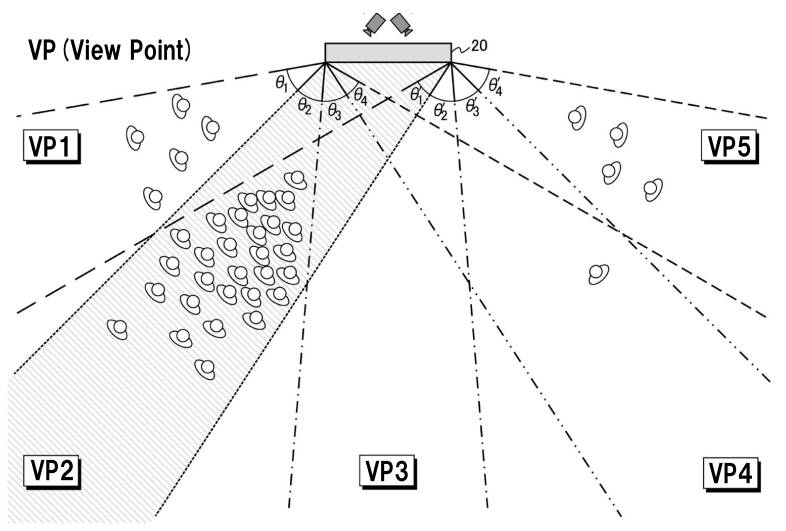

Figure 12: In a large field application, audiences can watch their preferred contents by self-movements.

Conventional digital signage does not provide audiences with freedom of choice because everyone has to see the same content. When viewed from the side that provides information to the general public, digital signage has no means to provide the appropriate information according to the attributes of the viewer. This situation is highly likely to be changed drastically by the proposed system. An individual can receive appropriate information everywhere, such as in a park, a stadium, a public hall, a station, and an airport, without needing to wear special glasses. Our aim is to promote the development of the system in terms of technology and application and it will also develop further needs of high resolution $\mathrm{LCD}$ over $4 \mathrm{~K}, 8 \mathrm{~K}$ and more. 


\section{ACKNOWLEDGMENTS}

This work was supported by JSPS KAKENHI Grant Numbers 25330244 and 25330440, and FUJITSU SOCIAL SCIENCE LABORATORY Ltd.

\section{REFERENCES}

[1] Antoine, G. Bois-clair. http://www . robertsimon.com/pdfs/boisclair_ portraits.pdf.

[2] FUJITSU-TEN. 2005. Press release: "two world first" - the eclipse car av \& navigation systems concept for the 39th tokyo motor show 2005. http://www.fujitsu-ten.com/release/ 2005/10/20051013_e.htm.

[3] Ishii, M. 2006. Spatial image by fractional view display. The Institute of Image Information and Television Engineers 30(58):33-38.

[4] Jagannatha, L., and Jawahar, C. 2005. Perspective correction methods for camera based document analysis. 148-154. In Proc. First Int. Workshop on Camera-based Document Analysis and Recognition.

[5] Jones, A.; Unger, J.; Nagano, K.; Busch, J.; Yu, X.; Peng, H.-Y.; Alexander, O.; Bolas, M.; and Debevec, P. 2015. An automultiscopic projector array for interactive digital humans. In ACM SIGGRAPH 2015 Emerging Technologies, SIGGRAPH '15, 6:1-6:1. New York, NY, USA: ACM.

[6] Mercedes-Benz. Mercedes-benz introduces new splitview comand display for the s-class. http://www. emercedesbenz.com/Dec08/ 10_001531_Mercedes_Benz_Introduces_ New_SplitView_COMAND_Display_For_The_ S_Class.html.

[7] Oliver, B., and Ramesh, R. 2005. Spatial Augmented Reality: Merging Real and Virtual Worlds.

[8] SHARP. Sharp dual directional viewing lcds. http://www.sharp-world.com/products / device/about/lcd/dual/index.html.

[9] Suzuki, H.; Hsieh, R.; and Shirai, A. 2014. Expixel: Pixelshader for multiplex-image hiding in consumer $3 \mathrm{~d}$ flat panels. In ACM SIGGRAPH 2014 Posters, SIGGRAPH '14, 63:1-63:1. New York, NY, USA: ACM.

[10] Vari-vue. Inventor of the lenticular imaging technique. http://www.didik.com/vv_his.htm.

[11] Yanaka, K. 2007. Integral photography suitable for small-lot production using mutually perpendicular lenticular sheets and fractional view. Proc. SPIE 6490:649016649016-9.

[12] Yanaka, K. 2008. Integral photography using hexagonal fly's eye lens and fractional view. Proc. SPIE 6803:68031K-68031K-8.

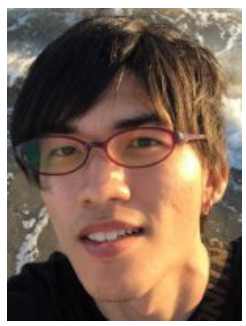

Hisataka SUZUKI is currently a Master Student studying entertainment system and virtual reality at Department of Electronics and Information Science at the Kanagawa Institute of Technology(KAIT) in Kanagawa(Japan). He joined Shirai Lab to research multiplex imagery technology. He was 3rd prize winner of ACM SIGGRAPH Student Research Competition in 2014. He was 3rd prize winner of CEDEC 2014 the largest game developper conference in Japan. He is going to join Sony Interactive Entertainment Inc. and may develop a new trends of entertainment.

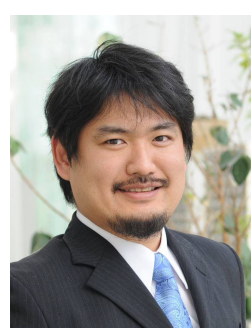

Akihiko SHIRAI was born in Yokohama, Japan, on March 13, 1973. He received his Bachelor of Engineering and Master of Engineering in photo engineering and image processing from Tokyo Polytechnic Institute in 19921998, and Ph.D. degrees in engineering from the Tokyo Institute of Technology (Tokyo Tech/TITECH) in 20012003. He moved to Kanagawa Institute of Technology, Associate Professor in Department of Information Media (2010). His research fields are entertainment VR system and its development.

Kazuhisa Yanaka received his Bach-

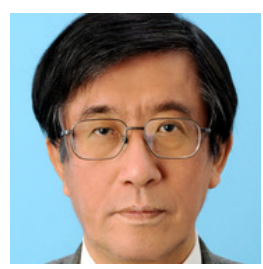
elor of Engineering, Master of Engineering, and $\mathrm{PhD}$ in Engineering from the University of Tokyo in 1977, 1979, and 1982, resepctively. He joined Electrical Communication Laboratories of NTT in 1982 and he was engaged in the research and development of visual communication systems. He moved to the Kanagawa Institute of Technology, Japan (1997). He is currently a professor at the Institute. His current main research fields are 3D display systems and optical illusion. 